\title{
On the asymptotic covariance of the multivariate empirical copula process
}

https://doi.org/10.1515/demo-2019-0015

Received February 24, 2019; revised June 18, 2019; second revision August 11, 2019; accepted August 11, 2019

Abstract: Genest and Segers (2010) gave conditions under which the empirical copula process associated with a random sample from a bivariate continuous distribution has a smaller asymptotic covariance than the standard empirical process based on a random sample from the underlying copula. An extension of this result to the multivariate case is provided.

Keywords: Empirical copula process, left-tail decreasing variable-by-variable, limiting covariance, rankbased inference

MSC: 60E15, 60F17, 62G05, 62G20, 62H05, 62H20

\section{Introduction}

Let $X_{1}, \ldots, X_{d}$ be random variables with joint cumulative distribution function $H$ and continuous univariate margins $F_{1}, \ldots, F_{d}$, respectively. From Sklar's Representation Theorem [24], there exists a unique $d$-variate copula $C$ such that, for all $x_{1}, \ldots, x_{d} \in \mathbb{R}$,

$$
H\left(x_{1}, \ldots, x_{d}\right)=C\left\{F_{1}\left(x_{1}\right), \ldots, F_{d}\left(x_{d}\right)\right\} .
$$

Inference about $C$ is central to copula modeling, which is now widespread. See, e.g., $[6,13,17,18,21]$ for surveys and applications in finance, insurance, risk management, and hydrology, among others.

Let $\left(X_{11}, \ldots, X_{1 d}\right), \ldots,\left(X_{n 1}, \ldots, X_{n d}\right)$ be a random sample from distribution $H$. If the margins $F_{1}, \ldots, F_{d}$ of $H$ are known, one can compute $U_{i j}=F_{j}\left(X_{i j}\right)$ for all $i \in\{1, \ldots, n\}$ and $j \in\{1, \ldots, d\}$. A consistent nonparametric estimator of $C$ is then given, for all $u_{1}, \ldots, u_{d} \in[0,1]$, by

$$
C_{n}\left(u_{1}, \ldots, u_{d}\right)=\frac{1}{n} \sum_{i=1}^{n} \mathbf{1}\left(U_{i 1} \leq u_{1}, \ldots, U_{i d} \leq u_{d}\right) .
$$

Indeed, standard asymptotic theory implies that, as $n \rightarrow \infty$, the empirical process $\mathbb{C}_{n}=\sqrt{n}\left(C_{n}-C\right)$ converges weakly in the space $\ell^{\infty}\left([0,1]^{d}\right)$ of bounded functions on $[0,1]^{d}$ endowed with the supremum norm $\|\cdot\|$. As stated, e.g., in [23], the limit is a centered Gaussian process $\mathbb{C}$ with continuous trajectories and covariance function given, for all $u_{1}, \ldots, u_{d}, v_{1}, \ldots, v_{d} \in[0,1]$, by

$$
\operatorname{cov}\left\{\mathbb{C}\left(u_{1}, \ldots, u_{d}\right), \mathbb{C}\left(v_{1}, \ldots, v_{d}\right)\right\}=C\left(u_{1} \wedge v_{1}, \ldots, u_{d} \wedge v_{d}\right)-C\left(u_{1}, \ldots, u_{d}\right) C\left(v_{1}, \ldots, v_{d}\right),
$$

where, for all $u, v \in[0,1], u \wedge v=\min (u, v)$.

\footnotetext{
*Corresponding Author: Christian Genest: Department of Mathematics and Statistics, McGill University, 805, rue Sherbrooke ouest, Montréal (Québec) Canada H3A 0B9

Mhamed Mesfioui: Département de mathématiques et d'informatique, Université du Québec à Trois-Rivières, C.P. 500, TroisRivières (Québec) Canada G9A 5H7

Johanna G. Nešlehová: Department of Mathematics and Statistics, McGill University, 805, rue Sherbrooke ouest, Montréal (Québec) Canada H3A 0B9
} 
In practice, however, the margins are typically unknown and hence the empirical distribution $C_{n}$ is an oracle estimator that cannot be computed. The standard solution to this problem is to resort to an analog of $C_{n}$ that relies on the marginal empirical distribution functions $F_{n 1}, \ldots, F_{n d}$ instead. For each $i \in\{1, \ldots, n\}$ and $j \in\{1, \ldots, d\}$, let $\hat{U}_{i j}=F_{n j}\left(X_{i j}\right)$. The empirical copula $\hat{C}_{n}$ is defined, for all $u_{1}, \ldots, u_{d} \in[0,1]$, by

$$
\hat{C}_{n}\left(u_{1}, \ldots, u_{d}\right)=\frac{1}{n} \sum_{i=1}^{n} \mathbf{1}\left(\hat{U}_{i 1} \leq u_{1}, \ldots, \hat{U}_{i d} \leq u_{d}\right) .
$$

The large-sample behavior of the process $\hat{\mathbb{C}}_{n}=\sqrt{n}\left(\hat{C}_{n}-C\right)$ has been studied since the mid-1970s; see, e.g., [10] and references therein. When it exists, the limit of this process involves the first partial derivatives of the copula $C$ defined, for all $j \in\{1, \ldots, d\}$ and $u_{1}, \ldots, u_{d} \in[0,1]$ with $0<u_{j}<1$, by

$$
\dot{C}_{j}\left(u_{1}, \ldots, u_{d}\right)=\frac{\partial}{\partial u_{j}} C\left(u_{1}, \ldots, u_{d}\right) .
$$

While these partial derivatives exist almost everywhere on $(0,1)^{d}$ as mentioned, e.g., in [20], this is not enough to ensure the weak convergence of $\widehat{\mathbb{C}}_{n}$. The following condition due to Segers [22] is sufficient.

Condition D (for derivatives): The copula $C$ is such that, for each $j \in\{1, \ldots, d\}, \dot{C}_{j}$ exists and is continuous on the set $\left\{\left(u_{1}, \ldots, u_{d}\right) \in[0,1]^{d}: 0<u_{j}<1\right\}$.

If $C$ satisfies Condition D, it follows from Proposition 3.1 in [22] that $\hat{\mathbb{C}}_{n}=\sqrt{n}\left(\hat{C}_{n}-C\right)$ converges weakly in $\ell^{\infty}\left([0,1]^{d}\right)$ to a $C$-pinned centered Gaussian process defined, for all $u_{1}, \ldots, u_{d} \in[0,1]^{d}$, by

$$
\hat{\mathbb{C}}\left(u_{1}, \ldots, u_{d}\right)=\mathbb{C}\left(u_{1}, \ldots, u_{d}\right)-\sum_{j=1}^{d} \dot{C}_{j}\left(u_{1}, \ldots, u_{d}\right) \mathbb{C}\left(u_{1 j}, \ldots, u_{d j}\right),
$$

where for arbitrary $j, k \in\{1, \ldots, d\}, u_{k j}=1+\left(u_{j}-1\right) \mathbf{1}(j=k)$ and $\dot{C}_{j}$ is extended to [0,1] $]^{d}$ as in [22].

The difference $\hat{\mathbb{C}}-\mathbb{C}$ is sometimes described as "the price to pay for not knowing the margins" but in dimension $d=2$, Genest and Segers [11] actually showed that, for all $u_{1}, u_{2}, v_{1}, v_{2} \in[0,1]$, one has

$$
\operatorname{cov}\left\{\hat{\mathbb{C}}\left(u_{1}, u_{2}\right), \hat{\mathbb{C}}\left(v_{1}, v_{2}\right)\right\} \leq \operatorname{cov}\left\{\mathbb{C}\left(u_{1}, u_{2}\right), \mathbb{C}\left(v_{1}, v_{2}\right)\right\}
$$

provided that $C$ satisfies Condition D and is left-tail decreasing (LTD) in the sense of Lehmann [16], i.e., when the maps $u_{1} \mapsto C\left(u_{1}, v_{2}\right) / u_{1}$ and $u_{2} \mapsto C\left(v_{1}, u_{2}\right) / u_{2}$ are non-increasing for all $v_{1}, v_{2} \in[0,1]$. When $C$ is LTD it is also positive quadrant dependent (PQD), i.e., for all $u_{1}, u_{2} \in[0,1], C\left(u_{1}, u_{2}\right) \geq u_{1} u_{2}$.

As a result, under the above conditions and if the margins are known, inference procedures based on $\hat{C}_{n}$ are asymptotically more efficient than their analogs based on $C_{n}$. This surprising fact has several consequences for inference in copula models, as highlighted by Genest and Segers [11]. However, these authors do not go beyond the bivariate case, except to state that their result holds for arbitrary $d \geq 2$ when $C$ is the independence copula defined, for all $u_{1}, \ldots, u_{d} \in[0,1]$, by $\Pi_{d}\left(u_{1}, \ldots, u_{d}\right)=u_{1} \times \cdots \times u_{d}$.

One possible extension of the finding of Genest and Segers [11] to arbitrary dimension is reported here. The main result, Theorem 1, is stated in Section 2 and proved in Section 3. It involves a new, multivariate extension of the LTD concept and an additional condition whose meaning and impact are further studied in Section 4. The cases of elliptical and Archimedean copulas are partially addressed in Sections 5 and 6, respectively. Closing remarks are given in Section 7.

In what follows, $\left(U_{1}, \ldots, U_{d}\right)$ stands for a vector of uniform random variables on $(0,1)$ with copula $C$ and, for any distinct $j, k \in\{1, \ldots, d\}, C_{j k}$ refers to the distribution of the pair $\left(U_{j}, U_{k}\right)$.

\section{Main result}

To extend the result of Genest and Segers [11] beyond the bivariate case, the following generalization of Lehmann's concept of left-tail decreasingness will be used. 
Definition 1. A d-variate copula $C$ is said to be left-tail decreasing variable-by-variable (LTD-VV) if, and only if, for all $j \in\{1, \ldots, d\}$ and any fixed values of $u_{1}, \ldots, u_{j-1}, u_{j+1}, \ldots, u_{d} \in[0,1]$, the map

$$
u_{j} \mapsto C\left(u_{1}, \ldots, u_{d}\right) / u_{j}=\operatorname{Pr}\left(U_{1} \leq u_{1}, \ldots, U_{d} \leq u_{d} \mid U_{j} \leq u_{j}\right)
$$

is non-increasing on $(0,1]$.

It is easily checked that when Condition D holds, the LTD-VV condition amounts to saying that, for all $j \in\{1, \ldots, d\}$ and $u_{1}, \ldots, u_{d} \in[0,1]$ with $0<u_{j}<1$,

$$
\dot{C}_{j}\left(u_{1}, \ldots, u_{d}\right) \leq C\left(u_{1}, \ldots, u_{d}\right) / u_{j} .
$$

The inequality remains valid for $u_{j}=1$ when $\dot{C}_{j}$ is extended to [0, 1] ${ }^{d}$ as in Eq. (2.2) of [22].

In dimension $d=2$, this concept coincides with the original definition of LTD due to Lehmann [16]. It further implies that $C$ is positively dependent in the following sense.

Lemma 1. Let $C$ be a d-variate LTD-VV copula. Then all lower-dimensional margins of $C$ of order 2 and above are LTD-VV and for all $u_{1}, \ldots, u_{d} \in[0,1], C\left(u_{1}, \ldots, u_{d}\right) \geq \Pi_{d}\left(u_{1}, \ldots, u_{d}\right)$. In particular, all bivariate margins of $C$ are LTD and $P Q D$.

Proof. To see that all lower-dimensional margins of $C$ are also LTD-VV, consider, without loss of generality, the distribution $C_{1 \ldots m}$ of $\left(U_{1}, \ldots, U_{m}\right)$. The fact that $C_{1 \ldots m}$ is LTD-VV follows at once from Definition 1 upon setting $u_{m+1}=\cdots=u_{d}=1$. Next note that $C\left(u_{1}, \ldots, u_{d}\right)=\Pi_{d}\left(u_{1}, \ldots, u_{d}\right)=0$ if at least one of the arguments equals 0 . To prove $C \geq \Pi_{d}$ on $(0,1]^{d}$, proceed by induction. If $C_{12}$ denotes the joint distribution of the pair $\left(U_{1}, U_{2}\right)$, then one has, for all $u_{1}, u_{2} \in(0,1], C_{12}\left(u_{1}, u_{2}\right) / u_{1} \geq C_{12}\left(1, u_{2}\right) / 1=u_{2}$ and, therefore, $C_{12}\left(u_{1}, u_{2}\right) \geq \Pi_{2}\left(u_{1}, u_{2}\right)$. Now suppose that $C_{1 \ldots m} \geq \Pi_{m}$. Then because $C_{1 \ldots(m+1)}$ is LTD-VV, one has, for all $u_{1}, \ldots, u_{m+1} \in(0,1], C_{1 \ldots(m+1)}\left(u_{1}, \ldots, u_{m+1}\right) \geq C_{1 \ldots m}\left(u_{1}, \ldots, u_{m}\right) \times u_{m+1}$. The induction assumption thus implies that $C_{1 \ldots(m+1)} \geq \Pi_{m+1}$ on $(0,1]^{d}$.

While Conditions D and LTD-VV suffice to establish the result of Genest and Segers [11] in dimension $d=2$, the following additional condition is needed for the multivariate extension proposed herein.

Condition CP (for conditional probabilities): For all $u_{1}, \ldots, u_{d} \in(0,1)$ and $j \in\{1, \ldots, d\}$,

$$
\sum_{k \neq j}\left\{\frac{C_{j k}\left(u_{j}, u_{k}\right)}{u_{k}}-u_{j}\right\} \leq \frac{C\left(u_{1}, \ldots, u_{d}\right)}{C\left(u_{1}, \ldots, u_{j-1}, 1, u_{j+1}, \ldots, u_{d}\right)}-u_{j},
$$

i.e., for $\left(U_{1}, \ldots, U_{d}\right)$ with distribution $C$,

$$
\begin{aligned}
\sum_{k \neq j}\left\{\operatorname{Pr}\left(U_{j} \leq u_{j} \mid U_{k} \leq u_{k}\right)\right. & \left.-\operatorname{Pr}\left(U_{j} \leq u_{j}\right)\right\} \\
& \leq \operatorname{Pr}\left(U_{j} \leq u_{j} \mid U_{1} \leq u_{1}, \ldots, U_{j-1} \leq u_{j-1}, U_{j+1} \leq u_{j+1}, \ldots, U_{d} \leq u_{d}\right)-\operatorname{Pr}\left(U_{j} \leq u_{j}\right) .
\end{aligned}
$$

The following result, which is this paper's main finding, is proved in Section 3.

Theorem 1. Let $C$ be an LTD-VV d-variate copula which satisfies Condition D. Suppose that $C$ also satisfies Condition $C P$. Then, for all $u_{1}, \ldots, u_{d}, v_{1}, \ldots, v_{d} \in[0,1]$,

$$
\operatorname{cov}\left\{\hat{\mathbb{C}}\left(u_{1}, \ldots, u_{d}\right), \hat{\mathbb{C}}\left(v_{1}, \ldots, v_{d}\right)\right\} \leq \operatorname{cov}\left\{\mathbb{C}\left(u_{1}, \ldots, u_{d}\right), \mathbb{C}\left(v_{1}, \ldots, v_{d}\right)\right\} .
$$

To see that Theorem 1 extends Proposition 1 in [11] to the $d$-variate case, it suffices to note that when $d=2$, Condition CP is void. Further note that when $C$ is LTD-VV, both the left- and right-hand side of inequality (3) are 
non-negative. For the left-hand side, this is because $C_{j k}$ is PQD for all distinct $j, k \in\{1, \ldots, d\}$ by Lemma 1 . The non-negativity of the right-hand side is equivalent to the inequality

$$
C\left(u_{1}, \ldots, u_{d}\right) / u_{j} \geq C\left(u_{1}, \ldots, u_{j-1}, 1, u_{j+1}, \ldots, u_{d}\right) / 1
$$

implied by the LTD-VV condition. This observation is used in the following example to show, among other things, that Theorem 1 also extends Proposition 4 of Genest and Segers [11] stating that inequality (4) holds for the independence copula $\Pi_{d}$.

Example 1. Consider an LTD-VV $d$-variate copula $C$ such that

$$
\forall_{j, k \in\{1, \ldots, d\}, j \neq k} \quad C_{j k}=\Pi_{j k} .
$$

Condition CP is then fulfilled because the left-hand side of inequality (3) vanishes while the right-hand side is non-negative because $C$ is LTD-VV, as stated in (5). Condition (6) is obviously satisfied by the independence copula $\Pi_{d}$. To give another example, consider the following extension of the Farlie-Gumbel-Morgenstern (FGM) copula defined, for all $u_{1}, \ldots, u_{d} \in[0,1]$, by

$$
C\left(u_{1}, \ldots, u_{d}\right)=\left(\prod_{j=1}^{d} u_{j}\right)\left\{1+\sum_{r=2}^{d} \sum_{\mathcal{A} \subseteq[d],|\mathcal{A}|=r} \theta_{\mathcal{A}} \prod_{j \in \mathcal{A}}^{d}\left(1-u_{j}\right)\right\},
$$

where $[d]=\{1, \ldots, d\}$ and the $2^{d}-d-1$ parameters are subject to constraints stated in Section 5.6 of [7]. It is immediate that the copulas in this class fulfill Condition D and that they are LTD-VV whenever all the parameters are non-negative. Furthermore, one has $C_{j k}=\Pi_{j k}$ for all distinct $j, k \in\{1, \ldots, d\}$ whenever $\theta_{\mathcal{A}}=0$ for all $\mathcal{A} \subseteq\{1, \ldots, d\}$ with $|\mathcal{A}|=2$. In such a case, it follows from Theorem 1 that inequality (4) holds for all $u_{1}, \ldots, u_{d}, v_{1}, \ldots, v_{d} \in[0,1]$.

Condition (6) is obviously not necessary. For instance, a simple calculation shows that in dimension $d=3$, the above FGM copula whose parameters are all equal to $\theta \in[0,1 / 2]$ satisfies Condition CP and hence all assumptions of Theorem 1.

Example 2. For some integer $m \in\{2, \ldots, d-2\}$, let $K$ and $L$ be $m$ - and $(d-m)$-variate copulas, respectively. Suppose that $K$ and $L$ are both LTD-VV and fulfill Condition CP. Then the $d$-variate copula $C$ defined, for all $u_{1}, \ldots, u_{d} \in[0,1]$, by

$$
C\left(u_{1}, \ldots, u_{d}\right)=K\left(u_{1}, \ldots, u_{m}\right) L\left(u_{m+1}, \ldots, u_{d}\right)
$$

is easily checked to be LTD-VV. Furthermore, $C$ satisfies Condition CP. Indeed, if $j \in\{1, \ldots, m\}$, then inequality (3) simplifies to

$$
\sum_{k \neq j, k \in[m]}\left\{\frac{K_{j k}\left(u_{j}, u_{k}\right)}{u_{k}}-u_{j}\right\} \leq \frac{K\left(u_{1}, \ldots, u_{m}\right)}{K\left(u_{1}, \ldots, u_{j-1}, 1, u_{j+1}, \ldots, u_{m}\right)}-u_{j} .
$$

which clearly holds because $K$ satisfies Condition CP. Similarly, if $j \in\{m+1, \ldots, d\}$, then the validity of inequality (3) stems from the fact that $L$ fulfills Condition CP.

Remark 1. From the calculations in Example 2, one can make the following observation. If a copula $C$ is LTD-VV and satisfies Condition CP, then so do all lower-dimensional margins of $C$ of order at least 2. To see this, consider, without loss of generality, the $m$-dimensional margin of $C$ obtained as the distribution of $\left(U_{1}, \ldots, U_{m}\right)$, where $\left(U_{1}, \ldots, U_{d}\right)$ has distribution $C$. The fact that it is LTD-VV follows at once from Lemma 1. Similarly, to see that Condition CP holds, choose an arbitrary $j \in\{1, \ldots, m\}$ and take the limit of both sides of inequality (3) as $u_{m+1}, \ldots, u_{d} \rightarrow 1$. The conclusion is then immediate.

From the proof of Theorem 1 given in Section 3, it transpires that the following condition, which is implied by Condition CP, is sufficient for inequality (4) to hold when $v_{1}=u_{1}, \ldots, v_{d}=u_{d}$, i.e., when one is only interested in comparing the asymptotic variances of the limiting processes $\widehat{\mathbb{C}}$ and $\mathbb{C}$. 
Condition V (for variances): For all $u_{1}, \ldots, u_{d} \in(0,1)$ and $j \in\{1, \ldots, d\}$,

$$
\sum_{k \neq j}\left\{\frac{C_{j k}\left(u_{j}, u_{k}\right)}{u_{k}}-u_{j}\right\} \leq 1-u_{j}
$$

i.e., for $\left(U_{1}, \ldots, U_{d}\right)$ with distribution $C$,

$$
\sum_{k \neq j}\left\{\operatorname{Pr}\left(U_{j} \leq u_{j} \mid U_{k} \leq u_{k}\right)-\operatorname{Pr}\left(U_{j} \leq u_{j}\right)\right\} \leq 1-u_{j} .
$$

Corollary 1. Let $C$ be an LTD-VV d-variate copula which satisfies Condition D. Suppose that $C$ also satisfies Condition $V$. Then, for all $u_{1}, \ldots, u_{d} \in[0,1]$,

$$
\operatorname{var}\left\{\hat{\mathbb{C}}\left(u_{1}, \ldots, u_{d}\right)\right\} \leq \operatorname{var}\left\{\mathbb{C}\left(u_{1}, \ldots, u_{d}\right)\right\} .
$$

\section{Proof of the main result}

Fix arbitrary $u_{1}, \ldots, u_{d}, v_{1}, \ldots, v_{d} \in[0,1]$ and for arbitrary $j, k \in\{1, \ldots, d\}$, set $u_{k j}=1+\left(u_{j}-1\right) \mathbf{1}(j=k)$ and $v_{k j}=1+\left(v_{j}-1\right) \mathbf{1}(j=k)$. In view of Eq. (1), the difference

$$
\operatorname{cov}\left\{\hat{\mathbb{C}}\left(u_{1}, \ldots, u_{d}\right), \hat{\mathbb{C}}\left(v_{1}, \ldots, v_{d}\right)\right\}-\operatorname{cov}\left\{\mathbb{C}\left(u_{1}, \ldots, u_{d}\right), \mathbb{C}\left(v_{1}, \ldots, v_{d}\right)\right\}
$$

can be expressed as

$$
\begin{gathered}
-\sum_{j=1}^{d} \dot{C}_{j}\left(v_{1}, \ldots, v_{d}\right) \operatorname{cov}\left\{\mathbb{C}\left(u_{1}, \ldots, u_{d}\right), \mathbb{C}\left(v_{1 j}, \ldots, v_{d j}\right)\right\} \\
-\sum_{j=1}^{d} \dot{C}_{j}\left(u_{1}, \ldots, u_{d}\right) \operatorname{cov}\left\{\mathbb{C}\left(v_{1}, \ldots, v_{d}\right), \mathbb{C}\left(u_{1 j}, \ldots, u_{d j}\right)\right\} \\
+\sum_{j=1}^{d} \sum_{k=1}^{d} \dot{C}_{j}\left(u_{1}, \ldots, u_{d}\right) \dot{C}_{k}\left(v_{1}, \ldots, v_{d}\right) \operatorname{cov}\left\{\mathbb{C}\left(u_{1 j}, \ldots, u_{d j}\right), \mathbb{C}\left(v_{1 k}, \ldots, v_{d k}\right)\right\} .
\end{gathered}
$$

Note that (8) vanishes if $u_{j}=0$ or $v_{j}=0$ for some $j \in\{1, \ldots, d\}$. Therefore, assume, without loss of generality, that $u_{1}, \ldots, u_{d}, v_{1}, \ldots, v_{d} \in(0,1]$. One can easily check that, for all $j \in\{1, \ldots, d\}$,

$$
\begin{aligned}
& \operatorname{cov}\left\{\mathbb{C}\left(u_{1}, \ldots, u_{d}\right), \mathbb{C}\left(v_{1 j}, \ldots, v_{d j}\right)\right\}=C\left(u_{1}, \ldots, u_{j} \wedge v_{j}, \ldots, u_{d}\right)-v_{j} C\left(u_{1}, \ldots, u_{d}\right), \\
& \operatorname{cov}\left\{\mathbb{C}\left(v_{1}, \ldots, v_{d}\right), \mathbb{C}\left(u_{1 j}, \ldots, u_{d j}\right)\right\}=C\left(v_{1}, \ldots, u_{j} \wedge v_{j}, \ldots, v_{d}\right)-u_{j} C\left(v_{1}, \ldots, v_{d}\right),
\end{aligned}
$$

and, for all $k \in\{1, \ldots, d\}$,

$$
\operatorname{cov}\left\{\mathbb{C}\left(u_{1 j}, \ldots, u_{d j}\right), \mathbb{C}\left(v_{1 k}, \ldots, v_{d k}\right)\right\}= \begin{cases}C_{j k}\left(u_{j}, v_{k}\right)-u_{j} v_{k} & \text { if } j \neq k, \\ u_{j} \wedge v_{j}-u_{j} v_{j} & \text { if } j=k .\end{cases}
$$

Therefore, the last summand in (8) can be decomposed as follows:

$$
\sum_{j=1}^{d} \dot{C}_{j}\left(u_{1}, \ldots, u_{d}\right) \dot{C}_{j}\left(v_{1}, \ldots, v_{d}\right)\left(u_{j} \wedge v_{j}-u_{j} v_{j}\right)+\sum_{j \neq k} \dot{C}_{j}\left(u_{1}, \ldots, u_{d}\right) \dot{C}_{k}\left(v_{1}, \ldots, v_{d}\right)\left\{C_{j k}\left(u_{j}, v_{k}\right)-u_{j} v_{k}\right\}
$$

In the light of these observations, one can write

$$
\operatorname{cov}\left\{\hat{\mathbb{C}}\left(u_{1}, \ldots, u_{d}\right), \hat{\mathbb{C}}\left(v_{1}, \ldots, v_{d}\right)\right\}-\operatorname{cov}\left\{\mathbb{C}\left(u_{1}, \ldots, u_{d}\right), \mathbb{C}\left(v_{1}, \ldots, v_{d}\right)\right\} \equiv \mathcal{S}_{1}+\mathcal{S}_{2}+\mathcal{S}_{3}+\mathcal{S}_{4}
$$


with

$$
\begin{aligned}
& \mathcal{S}_{1}=-\sum_{j=1}^{d} \dot{C}_{j}\left(v_{1}, \ldots, v_{d}\right)\left\{C\left(u_{1}, \ldots, u_{j} \wedge v_{j}, \ldots, u_{d}\right)-v_{j} C\left(u_{1}, \ldots, u_{d}\right)\right\}, \\
& \mathcal{S}_{2}=-\sum_{j=1}^{d} \dot{C}_{j}\left(u_{1}, \ldots, u_{d}\right)\left\{C\left(v_{1}, \ldots, u_{j} \wedge v_{j}, \ldots, v_{d}\right)-u_{j} C\left(v_{1}, \ldots, v_{d}\right)\right\}, \\
& \mathcal{S}_{3}=\sum_{j=1}^{d} \dot{C}_{j}\left(u_{1}, \ldots, u_{d}\right) \dot{C}_{j}\left(v_{1}, \ldots, v_{d}\right)\left(u_{j} \wedge v_{j}-u_{j} v_{j}\right), \\
& \mathcal{S}_{4}=\sum_{j \neq k} \dot{C}_{j}\left(u_{1}, \ldots, u_{d}\right) \dot{C}_{k}\left(v_{1}, \ldots, v_{d}\right)\left\{C_{j k}\left(u_{j}, v_{k}\right)-u_{j} v_{k}\right\} .
\end{aligned}
$$

It will first be shown that $\mathcal{S}_{1}+\mathcal{S}_{3} \leq 0$. Given that the copula $C$ is LTD-VV by assumption, inequality (2) holds for all $j \in\{1, \ldots, d\}$, and hence $\delta_{1}+\delta_{3}$ is bounded above by

$$
\sum_{j=1}^{d} \dot{C}_{j}\left(v_{1}, \ldots, v_{d}\right)\left[\left(u_{j} \wedge v_{j}\right)\left\{\frac{C\left(u_{1}, \ldots, u_{d}\right)}{u_{j}}-\frac{C\left(u_{1}, \ldots, u_{j} \wedge v_{j}, \ldots, u_{d}\right)}{u_{j} \wedge v_{j}}\right\}\right] .
$$

Furthermore, one has

$$
C\left(u_{1}, \ldots, u_{d}\right) / u_{j} \leq C\left(u_{1}, \ldots, u_{j} \wedge v_{j}, \ldots, u_{d}\right) /\left(u_{j} \wedge v_{j}\right)
$$

because $C$ is LTD-VV by assumption. It then follows from (9) that $\delta_{1}+\delta_{3} \leq 0$.

Next, it will be shown that $S_{2}+S_{4} \leq 0$. This is where Condition CP plays a role. Using again the inequality $\dot{C}_{k}\left(v_{1}, \ldots, v_{d}\right) \leq C\left(v_{1}, \ldots, v_{d}\right) / v_{k}$, which is valid for all $k \in\{1, \ldots, d\}$, one finds that

$$
\begin{aligned}
\mathcal{S}_{2}+\mathcal{S}_{4} \leq \sum_{j=1}^{d} \dot{C}_{j}\left(u_{1}, \ldots, u_{d}\right)\left[-C\left(v_{1}, \ldots, u_{j} \wedge v_{j}, \ldots, v_{d}\right)+u_{j} C\left(v_{1}, \ldots, v_{d}\right)\right. \\
\left.+\sum_{k \neq j}\left[\frac{C\left(v_{1}, \ldots, v_{d}\right)}{v_{k}}\left\{C_{j k}\left(u_{j}, v_{k}\right)-u_{j} v_{k}\right\}\right]\right] .
\end{aligned}
$$

It will be shown that for every $j \in\{1, \ldots, d\}$, one has

$$
\sum_{k \neq j} \frac{C\left(v_{1}, \ldots, v_{d}\right)}{v_{k}}\left\{C_{j k}\left(u_{j}, v_{k}\right)-u_{j} v_{k}\right\} \leq C\left(v_{1}, \ldots, u_{j} \wedge v_{j}, \ldots, v_{d}\right)-u_{j} C\left(v_{1}, \ldots, v_{d}\right) .
$$

If $C\left(v_{1}, \ldots, v_{d}\right)=0$, this inequality trivially holds. If $C\left(v_{1}, \ldots, v_{d}\right)>0$, then it is equivalent to

$$
\sum_{k \neq j}\left\{\frac{C_{j k}\left(u_{j}, v_{k}\right)}{v_{k}}-u_{j}\right\} \leq \frac{C\left(v_{1}, \ldots, u_{j} \wedge v_{j}, \ldots, v_{d}\right)}{C\left(v_{1}, \ldots, v_{d}\right)}-u_{j} .
$$

To see that this inequality holds when Condition CP is satisfied, and in view of Remark 1 , it suffices to show that

$$
\frac{C\left(v_{1}, \ldots, u_{j} \wedge v_{j}, \ldots, v_{d}\right)}{C\left(v_{1}, \ldots, v_{d}\right)} \geq \frac{C\left(v_{1}, \ldots, u_{j}, \ldots, v_{d}\right)}{C\left(v_{1}, \ldots, v_{j-1}, 1, v_{j+1}, \ldots, v_{d}\right)} .
$$

This is clearly true when $u_{j} \geq v_{j}$, because the left-hand side then equals 1 while the right-hand side is always at most 1 . If $u_{j}<v_{j}$, then the inequality holds because the numerators coincide while

$$
C\left(v_{1}, \ldots, v_{d}\right) \leq C\left(v_{1}, \ldots, v_{j-1}, 1, v_{j+1}, \ldots, v_{d}\right) .
$$

This completes the proof of Theorem 1. 


\section{Consequences of Conditions CP and V}

Condition $\mathrm{CP}$ is generally difficult to verify. To simplify matters, it would thus be tempting to assume that it holds term by term, i.e., that for all distinct $j, k \in\{1, \ldots, d\}$ and all $u_{1}, \ldots, u_{d} \in(0,1)$, one has

$$
\frac{C_{j k}\left(u_{j}, u_{k}\right)}{u_{k}} \leq \frac{1}{d-1} \frac{C\left(u_{1}, \ldots, u_{d}\right)}{C\left(u_{1}, \ldots, u_{j-1}, 1, u_{j+1}, \ldots, u_{d}\right)}+\frac{d-2}{d-1} u_{j} .
$$

If $C$ is LTD-VV and $C_{j k}=\Pi_{j k}$, i.e., when $C_{j k}$ is the independence copula, this inequality reduces to (5), which has been seen to hold. Therefore, all LTD-VV $d$-variate copulas $C$ with the property (6) satisfy inequality (10) and hence, a fortiori, Condition CP, as discussed in Example 1. Unfortunately, it turns out that inequality (10) also implies (6) when $C$ is LTD-VV, and hence the term-by-term version of Condition CP is not useful in practice. This is shown next.

Proposition 1. Let $C$ be an $L T D$-VV d-variate copula satisfying inequality (10) for all distinct $j, k \in\{1, \ldots, d\}$ and all $u_{1}, \ldots, u_{d} \in(0,1)$. Then $C_{j k}=\Pi_{j k}$ for all distinct $j, k \in\{1, \ldots, d\}$.

Proof. Fix distinct $j, k \in\{1, \ldots, d\}$ and arbitrary $u_{j}, u_{k} \in(0,1)$. Let $u_{\ell} \rightarrow 1$ for all $\ell \in\{1, \ldots, d\} \backslash\{j, k\}$. Then inequality (10) reduces to

$$
\frac{C_{j k}\left(u_{j}, u_{k}\right)}{u_{k}} \leq \frac{1}{d-1} \frac{C_{j k}\left(u_{j}, u_{k}\right)}{u_{k}}+\frac{d-2}{d-1} u_{j},
$$

which is equivalent to the requirement that $C_{j k}\left(u_{j}, u_{k}\right) \leq u_{j} u_{k}$. However, given that $C$ is LTD-VV by assumption, one also has $C_{j k}\left(u_{j}, u_{k}\right) \geq u_{j} u_{k}$ by Lemma 1 . Considering that the choices of $u_{j}$ and $u_{k}$ were arbitrary, it follows that $C_{j k}=\Pi_{j k}$ for any distinct $j, k \in\{1, \ldots, d\}$, as claimed.

One can shed further light on the requirements imposed by Condition CP by considering the special case of Condition V. First, here is an equivalent formulation of this condition for LTD-VV copulas.

Proposition 2. Let $C$ be an LTD-VV d-variate copula satisfying Condition D. For all distinct $j, k \in\{1, \ldots, d\}$ and $u \in(0,1)$, let $\stackrel{\circ}{C}_{j k}(u)=\lim _{v \downarrow 0} C_{j k}(u, v) / v$. Then $C$ satisfies Condition $V$ if, and only if,

$$
\forall u \in(0,1) \quad \sum_{k \neq j} \stackrel{\circ}{C}_{j k}(u) \leq 1+(d-2) u .
$$

Proof. For all distinct $j, k \in\{1, \ldots, d\}, C_{j k}$ is LTD by assumption, i.e., the map $v \mapsto C_{j k}(u, v) / v$ is nonincreasing whatever the value of $u \in(0,1)$. Therefore, for arbitrary $u_{1}, \ldots, u_{d} \in(0,1)$,

$$
C_{j k}\left(u_{j}, u_{k}\right) / u_{k} \leq \lim _{u \downarrow 0} C_{j k}\left(u_{j}, u\right) / u=\stackrel{\circ}{C}_{j k}\left(u_{j}\right),
$$

and hence inequality (7) holds whenever

$$
\sum_{k \neq j}\left\{\stackrel{\circ}{C}_{j k}(u)-u\right\} \leq 1-u,
$$

which is equivalent to inequality (11). However, it is also true that inequality (7) implies inequality (11) by taking the limit as $u_{k} \downarrow 0$ for all $k \neq j$ in (7).

For the rest of this section, the discussion is limited, for simplicity, to the important special case of exchangeable copulas, whose definition is recalled below for convenience.

Definition 2. A copula $C$ is said to be exchangeable if, and only if, for all $u_{1}, \ldots, u_{d} \in[0,1]$ and every permutation $\pi$ on $\{1, \ldots, d\}$, one has $C\left(u_{1}, \ldots, u_{d}\right)=C\left(u_{\pi(1)}, \ldots, u_{\pi(d)}\right)$. In that case, all bivariate margins of $C$ are identical and are hereafter denoted $B_{2}$. 
A first constraint imposed by Condition V on an exchangeable LTD-VV copula $C$ is that its common bivariate margin $B_{2}$ is in a small neighborhood of the bivariate independence copula $\Pi_{2}$. This distance is measured in terms of the supremum norm, defined by

$$
\left\|B_{2}-\Pi_{2}\right\|=\sup _{u, v \in[0,1]}\left|B_{2}(u, v)-\Pi_{2}(u, v)\right| .
$$

Proposition 3. Let $C$ be a d-variate exchangeable copula with common bivariate margin $B_{2}$. If $C$ is $L T D$-VV and satisfies Condition $V$, then $\left\|B_{2}-\Pi_{2}\right\| \leq 1 /(d-1)$.

Proof. For arbitrary $u, v \in(0,1)$, set $v_{j}=u$ and $v_{k}=v$ for all $k \neq j$ in inequality (7). One then has

$$
\sum_{k \neq j}\left\{C_{j k}(u, v)-u v\right\} \leq v(1-u) \leq 1
$$

and hence

$$
\frac{1}{d-1} \sum_{k \neq j} C_{j k}(u, v)-u v \leq \frac{1}{d-1} .
$$

Invoking the fact that $C_{j k}=B_{2}$ for all distinct $j, k \in\{1, \ldots, d\}$, this inequality reduces to $B_{2}(u, v)-u v \leq$ $1 /(d-1)$. Given that $C$ is LTD-VV by assumption, $B_{2}$ is PQD, and hence $B_{2}(u, v)-u v \geq 0$. This yields the desired conclusion.

Proposition 3 implies that in the case of exchangeable copulas, the conditions under which Theorem 1 was established can only hold in a small (LTD-VV) neighborhood around independence. An illustration of Proposition 3 is provided by the FGM copulas defined in Example 1 with the additional property that $\theta_{\mathcal{A}}=0$ if $|\mathcal{A}|=2$ and $\theta_{\mathcal{A}}=\theta \in(0,1)$ if $|\mathcal{A}| \geq 3$. In that case, one actually has $\left\|B_{2}-\Pi_{2}\right\|=0$.

Next consider the tail behavior of the common bivariate margin $B_{2}$ of $C$, as measured by the lower- and upper-tail dependence indices [13]. When they exist, these coefficients are respectively defined by

$$
\lambda_{L}=\lim _{t \downarrow 0} B_{2}(t, t) / t \quad \text { and } \quad \lambda_{U}=2-\lim _{t \uparrow 1}\left\{1-B_{2}(t, t)\right\} /(1-t) .
$$

Proposition 4. Let $C$ be a d-variate exchangeable copula with common bivariate margin $B_{2}$. Suppose further that Condition $V$ holds for $C$. If they exist, the lower-and upper-tail indices then both satisfy $\lambda_{L} \leq 1 /(d-1)$ and $\lambda_{U} \leq 1 /(d-1)$.

Proof. For arbitrary $t \in(0,1)$, set $u_{1}=\cdots=u_{d}=t$ in inequality (7). Upon substitution, one then gets

$$
\sum_{k \neq j} C_{j k}(t, t) / t \leq 1+(d-2) t,
$$

and hence upon taking the limit as $t \downarrow 0$, one finds that the sum of the lower-tail coefficients must be at most 1. Now exploiting the fact that $C_{j k}=B_{2}$ for all distinct $j, k \in\{1, \ldots, d\}$, one deduces that

$$
(d-1) B_{2}(t, t) / t \leq 1+(d-2) t,
$$

which implies that

$$
\lambda_{L}=\lim _{t \downarrow 0} B_{2}(t, t) / t \leq 1 /(d-1),
$$

as claimed. Similarly, one can deduce from inequality (12) that

$$
\lambda_{U}=2+\lim _{t \uparrow 1} \frac{B_{2}(t, t)-1}{1-t} \leq 2+\lim _{t \uparrow 1} \frac{(d-2) t^{2}+t-(d-1)}{(d-1)(1-t)}=\frac{1}{d-1},
$$

as claimed.

Thus, in view of Proposition 4, the conditions under which Theorem 1 was established also restrict considerably the degree of tail dependence that a $d$-variate exchangeable LTD-VV copula could have if it also satisfies Condition CP and hence Condition V. 


\section{The case of elliptical copulas}

Suppose that a random vector $Z=\left(Z_{1}, \ldots, Z_{d}\right)$ can be expressed in the form

$$
Z \stackrel{d}{=} \mu+R A T,
$$

where $\stackrel{d}{=}$ denotes equality in distribution, $R$ is a strictly positive random variable, $A$ is a $d \times d$ matrix of constants, and $T=\left(T_{1}, \ldots, T_{d}\right)$ is a random vector which is independent of $R$ and uniformly distributed on the unit sphere $\mathcal{T}_{d}=\left\{\left(t_{1}, \ldots, t_{d}\right) \in \mathbb{R}^{d}: t_{1}^{2}+\cdots+t_{d}^{2}=1\right\}$. The vector $Z$ then has an elliptical distribution with mean $\mu$ and dispersion matrix $\Sigma=A A^{\top}$, and its underlying copula is called elliptical.

All elliptical copulas are copulas of random vectors which admit a stochastic representation of the form (13) with $\mu=0$ and $\Sigma=\left(\rho_{j k}\right)$ with $\rho_{j j}=1$ for all $j \in\{1, \ldots, d\}$. Of frequent use in practice are the elliptical copulas associated with the multivariate Gaussian and Student $t$ distributions. For background concerning elliptical distributions and copulas, see, e.g., $[8,9,25]$.

Given any $Z=\left(Z_{1}, \ldots, Z_{d}\right)$ of the form (13) with $\mu=0$, and for any distinct $j, k \in\{1, \ldots, d\}$, let

$$
\beta\left(Z_{j}, Z_{k}\right)=-1+4 \operatorname{Pr}\left(Z_{j} \leq 0, Z_{k} \leq 0\right) .
$$

As detailed in [12], $\beta\left(Z_{j}, Z_{k}\right)$ is the medial correlation coefficient of the pair $\left(Z_{j}, Z_{k}\right)$, also known as Blomqvist's beta, and it coincides with the value of Kendall's tau for that pair. From formulas (5) and (6) in [12], one has in fact

where $\rho_{j k}$ is the $(j, k)$ th entry of the matrix $\Sigma$.

$$
\beta\left(Z_{j}, Z_{k}\right)=\tau\left(Z_{j}, Z_{k}\right)=\frac{2}{\pi} \arcsin \left(\rho_{j k}\right),
$$

Proposition 5. Let $C$ be the unique copula of a random vector $Z=\left(Z_{1}, \ldots, Z_{d}\right)$ having a non-degenerate elliptical distribution. Suppose that there exist distinct indices $j, k, \ell \in\{1, \ldots, d\}$ such that $\beta\left(Z_{j}, Z_{k}\right)>0$, $\beta\left(Z_{j}, Z_{\ell}\right)>0$, and $\beta\left(Z_{k}, Z_{\ell}\right)>0$. Then the copula $C$ fails to satisfy Condition $C P$.

Proof. The argument hinges on the fact that inequality (3) holds if, and only if, for all $j \in\{1, \ldots, d\}$ and $z_{1}, \ldots, z_{d} \in \mathbb{R}$,

$$
\begin{aligned}
\sum_{k \neq j}\left\{\operatorname{Pr}\left(Z_{j} \leq z_{j} \mid Z_{k} \leq z_{k}\right)\right. & \left.\operatorname{Pr}\left(Z_{j} \leq z_{j}\right)\right\} \\
& \leq \operatorname{Pr}\left(Z_{j} \leq z_{j} \mid Z_{1} \leq z_{1}, \ldots, Z_{j-1} \leq z_{j-1}, Z_{j+1} \leq z_{j+1}, \ldots, Z_{d} \leq z_{d}\right)-\operatorname{Pr}\left(Z_{j} \leq z_{j}\right) .
\end{aligned}
$$

In view of Remark 1, it suffices to consider the case $d=3$. It will be shown that inequality (14) actually fails when $z_{1}=z_{2}=z_{3}=0$. The proof exploits the fact stated, e.g., in Chapter 45 of [14], that

$$
-1+8 \operatorname{Pr}\left(Z_{1} \leq 0, Z_{2} \leq 0, Z_{3} \leq 0\right)=\beta\left(Z_{1}, Z_{2}\right)+\beta\left(Z_{1}, Z_{3}\right)+\beta\left(Z_{2}, Z_{3}\right) .
$$

This expression is often attributed to Cramér [5] in the Gaussian case, but it remains valid for all nondegenerate elliptical distributions, as pointed out in Section 2.7 of [8].

Fixing $j=1$ in (14), and using the above relation together with the obvious fact that $\operatorname{Pr}\left(Z_{j} \leq 0\right)=1 / 2$, one can easily see that inequality (14) holds at $z_{1}=z_{2}=z_{3}=0$ if, and only if,

$$
\beta\left(Z_{2}, Z_{3}\right)\left\{\beta\left(Z_{1}, Z_{2}\right)+\beta\left(Z_{1}, Z_{3}\right)\right\} \leq 0 .
$$

Similar arguments can be made fixing either $j=2$ or $j=3$. It follows that the above inequality must hold, but also the following two:

$$
\beta\left(Z_{1}, Z_{3}\right)\left\{\beta\left(Z_{1}, Z_{2}\right)+\beta\left(Z_{2}, Z_{3}\right)\right\} \leq 0, \quad \beta\left(Z_{1}, Z_{2}\right)\left\{\beta\left(Z_{1}, Z_{3}\right)+\beta\left(Z_{2}, Z_{3}\right)\right\} \leq 0 .
$$

As these three inequalities cannot hold simultaneously when $\beta\left(Z_{1}, Z_{2}\right), \beta\left(Z_{1}, Z_{3}\right)$, and $\beta\left(Z_{2}, Z_{3}\right)$ are all strictly positive, one can conclude that inequality (14) fails at $z_{1}=z_{2}=z_{3}=0$ and hence in general.

In view of Proposition 5, the conditions of Theorem 1 are thus too strong to encompass elliptical copulas, unless perhaps in special cases when the dispersion matrix is sparse. This is not to say that inequality (4) fails for all these copulas, however. 


\section{The case of Archimedean copulas}

Recall from [19] that the map defined, for all $u_{1}, \ldots, u_{d} \in(0,1)$, by

$$
C_{\psi}\left(u_{1}, \ldots, u_{d}\right)=\psi\left\{\phi\left(u_{1}\right)+\cdots+\phi\left(u_{d}\right)\right\} .
$$

is an Archimedean copula with generator $\psi:[0, \infty) \rightarrow[0,1]$ if, and only if, $\psi$ is $d$-monotone and such that $\psi(0)=1$ and $\psi(x) \rightarrow 0$ as $x \rightarrow \infty$. Here, $\phi=\psi^{-1}, \psi(\infty)=0$, and $\phi(0)=\inf \{x \in[0, \infty): \psi(x)=0\}$.

As the applicability of Theorem 1 is of current interest, attention can immediately be restricted to the case where the Archimedean generator $\psi$ is strict, i.e., $\phi(0)=\infty$. This is because if $\phi(0)<\infty$, then $C_{\psi} \nexists \Pi_{d}$ and hence $C_{\psi}$ is not LTD-VV either by Lemma 1.

Lemma 2. Let $C$ be a d-variate Archimedean copula with a strict generator $\psi$. Then $C$ is LTD-VV if, and only if, $\psi$ is log-convex.

Proof. Let $C=C_{\psi}$ be a $d$-variate Archimedean copula with strict generator $\psi$. In view of Lemma 1, if $C$ is LTD-VV, then so are its bivariate margins, which are bivariate Archimedean copulas with generator $\psi$. From Proposition 3 in [1], such a bivariate copula is LTD if, and only if, $\ln (\psi)$ is convex. Reciprocally, if $\ln (\psi)$ is convex, then for all $x, y, z \in(0, \infty)$ with $y<z$, one has

$$
\psi(x+y) / \psi(y) \leq \psi(x+z) / \psi(z) .
$$

In other words, the map $y \mapsto \psi(x+y) / \psi(y)$ is non-decreasing on $(0, \infty)$ for all $x>0$. Now fix $j \in\{1, \ldots, d\}$ and set $x=\sum_{k \neq j} \phi\left(u_{k}\right), y=\phi\left(u_{j}\right)$ and $z=\phi\left(u_{j}^{\prime}\right)$ for any $u_{1}, \ldots, u_{d}, u_{j}^{\prime} \in(0,1)$ with $u_{j}>u_{j}^{\prime}$. Given that $\phi$ is non-increasing, one has $\psi(y)=u_{j}>\psi(z)=u_{j}^{\prime}$. Eq. (15) then implies that the map $u_{j} \mapsto C\left(u_{1}, \ldots, u_{d}\right) / u_{j}$ is non-increasing on $(0,1)$, and hence by continuity on $(0,1]$. This is precisely the definition of the LTD-VV concept.

Remark 2. As a consequence of Lemma 2, a $d$-variate Archimedean copula with a completely monotone generator $\psi$ is necessarily LTD-VV given that $\psi$ is log-convex in this case; see the proof of Corollary 4.6.3 in [20]. Also, for Archimedean copulas with strict generators, the LTD-VV property is equivalent to the concept of multivariate left-tail decreasingness (MLTD) which is introduced in [4] and recalled here for convenience. A vector $\left(U_{1}, \ldots, U_{d}\right)$ of uniform random variables on $(0,1)$ or its copula $C$ is said to be left-tail decreasing in sequence (LTDS) if, for all $j \in\{1, \ldots, d-1\}$ and $u_{j+1} \in(0,1)$, the map

$$
\left(u_{1}, \ldots, u_{j}\right) \mapsto \operatorname{Pr}\left(U_{j+1} \leq u_{j+1} \mid U_{1} \leq u_{1}, \ldots, U_{j} \leq u_{j}\right)
$$

is non-increasing in each of its components. The copula $C$ is further said to be multivariate left-tail decreasing (MLTD) if $\left(U_{\pi(1)}, \ldots, U_{\pi(d)}\right)$ is LTDS for all permutations $\pi$ on $\{1, \ldots, d\}$. In view of Lemma 2 , these three notions, i.e., LTD-VV, LTDS and MLTD, are equivalent for Archimedean copulas, because the latter are exchangeable.

The following negative result shows that once an Archimedean copula is LTD-VV, Condition CP cannot hold unless $C=\Pi_{d}$ or $d=2$.

Proposition 6. Let $C$ be a d-variate Archimedean copula with a log-convex, strict generator $\psi$. Then $C$ is LTD$V V$ but Condition CP does not hold, unless $d=2$ or $C=\Pi_{d}$.

Proof. First note that for any fixed $z \in[0, \infty), \psi(z+x) / \psi(x) \in[\psi(z), 1]$ for all $x \in[0, \infty)$, owing to the fact that $\psi$ is strictly decreasing. Because the map $x \mapsto \psi(z+x) / \psi(x)$ is non-decreasing by assumption, there exists $L_{z} \in[\psi(z), 1]$ such that

$$
\lim _{x \rightarrow \infty} \psi(z+x) / \psi(x)=L_{z} .
$$


Now suppose that Condition CP holds and fix an arbitrary $j \in\{1, \ldots, d\}$. For any $v_{1}, \ldots, v_{d} \in(0,1)$, set $z=\phi\left(v_{j}\right)$ and $x_{k}=\phi\left(v_{k}\right)$ for $k \neq j \in\{1, \ldots, d\}$. Then inequality (3) can be rewritten as

$$
\sum_{k \neq j}\left\{\psi\left(z+x_{k}\right) / \psi\left(x_{k}\right)-\psi(z)\right\} \leq \psi\left(z+\sum_{k \neq j} x_{k}\right) / \psi\left(\sum_{k \neq j} x_{k}\right)-\psi(z) .
$$

Letting $x_{k} \rightarrow \infty$ for all $k \in\{1, \ldots, d\} \backslash\{j\}$ on both sides of this inequality leads to

$$
(d-1)\left\{L_{z}-\psi(z)\right\} \leq L_{z}-\psi(z) .
$$

When $d \neq 2$ this is possible only if $L_{z}=\psi(z)$, which implies that the map $x \mapsto \psi(z+x) / \psi(x)$ is constant. Because $v_{j}$, and hence $z$, was arbitrary, this means that $\psi(z+x)=\psi(x) \psi(z)$ for all $x, z \in[0, \infty)$. The only solution to this functional equation is the independence generator given by $\psi(x)=e^{-x}$ for all $x \in[0, \infty)$. This concludes the argument.

Although Proposition 6 implies that Condition CP cannot hold for LTD-VV Archimedean copulas other than independence, there are non-trivial examples of such copulas that do satisfy Condition V. Focusing, without loss of generality, on the case $d>2$, note first that when $C$ is Archimedean with generator $\psi$, the latter is differentiable because it is at least 3-monotone. Inequality (11) then reduces to the requirement that, for all $z \in(0, \infty)$,

$$
\lim _{x \rightarrow \infty} \frac{\psi^{\prime}(x+z)}{\psi^{\prime}(x)} \leq \frac{d-2}{d-1} \psi(z)+\frac{1}{d-1} .
$$

As the following examples show, whether (16) holds or not depends on the extremal properties of $\psi$. Recall that $\psi$ is also a survival function on $[0, \infty)$, and as such may be in the maximum domain of either the Weibull, Fréchet or Gumbel distributions; see, e.g., [15]. Essentially all well-known Archimedean generators fall in either one of these three classes, as shown in [3]. The Weibull class is not interesting here, because when $\psi$ is in the Weibull domain, $\phi(0)<\infty$ and so the resulting Archimedean copula is not LTD-VV. The other two cases are elaborated on in the examples below. These show in particular that if Condition $\mathrm{V}$ is to hold, $\psi$ can only be light-tailed.

Example 3. Suppose that $\psi$ is a differentiable Archimedean generator in the maximum domain of attraction of the Fréchet distribution. Then $\psi \in \mathcal{R}_{-\alpha}$, i.e., $\psi$ is regularly varying with index $-\alpha$ for some $\alpha \in(0, \infty)$. This means that, for all $x \in(0, \infty)$,

$$
\lim _{t \rightarrow \infty} \psi(t x) / \psi(t)=x^{-\alpha},
$$

the convergence being locally uniform in $t$. A prime example is the Clayton generator defined, for all $x \in$ $[0, \infty)$, by $\psi=(1+x / \alpha)^{-\alpha}$ for some $\alpha \in(0, \infty)$.

When $\psi \in \mathcal{R}_{-\alpha}$, the lower-tail dependence coefficient of the Archimedean copula $C_{\psi}$ is $2^{-\alpha}>0$ as stated, e.g., in Corollary 2 of [15]. Thus from Proposition 4, $\psi$ could only satisfy Condition (16) if $\alpha \geq \log _{2}(d-1)$. As it happens, however, inequality (16) is never satisfied in this case. For, if $\psi \in \mathcal{R}_{-\alpha}$, then $-\psi^{\prime} \in \mathcal{R}_{-\alpha-1}$ as a consequence of the monotone density theorem; see, e.g., Theorem 1.7.2 in [2]. It follows at once that

$$
\lim _{x \rightarrow \infty} \psi^{\prime}(x+z) / \psi^{\prime}(x)=\lim _{x \rightarrow \infty} \psi^{\prime}\{x(1+z / x)\} / \psi^{\prime}(x)=1 .
$$

Therefore, the left-hand side of (16) is then equal to 1 uniformly in $z \in(0, \infty)$, while the right-hand side is strictly smaller than 1 for all $z \in(0, \infty)$.

Example 4. Suppose that $\psi$ is a strict and differentiable generator which is in the maximum domain of attraction of the Gumbel distribution. From the proof of Theorem 1(c) in [15], it follows that $-1 / \psi^{\prime}$ is $\Gamma$-varying, i.e., there exists a function $a:(0, \infty) \rightarrow(0, \infty)$ such that, for all $z \in \mathbb{R}$,

$$
\lim _{x \rightarrow \infty} \psi^{\prime}\{x+z a(x)\} / \psi^{\prime}(x)=e^{-z},
$$

where $-\psi / \psi^{\prime}$ is a possible choice for $a$. From Lemma $2, C_{\psi}$ is LTD-VV if, and only if, $-\psi^{\prime} / \psi$ is non-increasing. Consequently, $a(x) \rightarrow a_{0} \in(0, \infty]$ as $x \rightarrow \infty$. Two cases can be distinguished. 
Suppose $a_{0}=\infty$. Given that $\psi^{\prime}$ is monotone, one must have

$$
\lim _{x \rightarrow \infty} \psi^{\prime}(x+z) / \psi^{\prime}(x)=\lim _{x \rightarrow \infty} \psi^{\prime}[x+a(x)\{z / a(x)\}] / \psi^{\prime}(x)=1,
$$

and hence inequality (16) does not hold for the same reasons as in Example 3.

Suppose that $a_{0}<\infty$. Then

$$
\lim _{x \rightarrow \infty} \psi^{\prime}(x+z) / \psi^{\prime}(x)=\lim _{x \rightarrow \infty} \psi^{\prime}[x+a(x)\{z / a(x)\}] / \psi^{\prime}(x)=e^{-z / a_{0}},
$$

and inequality (16) is then fulfilled whenever, for all $u \in[0,1]$,

$$
\exp \left\{-\phi(u) / a_{0}\right\} \leq \frac{d-2}{d-1} u+\frac{1}{d-1} .
$$

A family for which inequality (17) holds for certain parameter values is the multivariate Ali-Mikhail-Haq copula with generator defined, for all $x \in[0, \infty)$ and $\theta \in[0,1]$, by $\psi(x)=(1-\theta) /\left(e^{x}-\theta\right)$. Here,

$$
\lim _{x \rightarrow \infty}-\frac{\psi(x)}{\psi^{\prime}(x)}=\frac{\left(e^{x}-\theta\right)^{2}}{e^{x}\left(e^{x}-\theta\right)}=a_{0}=1 .
$$

Condition (17) then reduces to

$$
\frac{u}{1-\theta(1-u)} \leq \frac{d-2}{d-1} u+\frac{1}{d-1} \quad \Leftrightarrow \quad \frac{(1-u)[1-\theta\{1+(d-2) u\}]}{1-\theta(1-u)} \geq 0 .
$$

Thus inequality (17) holds, and hence inequality (16) is fulfilled, when $1-\theta\{1+(d-2) u\} \geq 0$ for all $u \in[0,1]$, which happens whenever $\theta \leq 1 /(d-1)$. Given that the generator of the Ali-Mikhail-Haq is the Laplace transform of a geometric random variable with success probability $\theta \in(0,1)$, it is completely monotone. As such it is automatically log-convex and hence this copula is LTD-VV. Therefore, the conditions of Corollary 1 are fulfilled for the Ali-Mikhail-Haq copula when $\theta \in[0,1 /(d-1)]$.

\section{Concluding remarks}

In this paper, conditions were identified under which the empirical copula process associated with a random sample from a $d$-variate continuous distribution has a smaller asymptotic covariance than the standard empirical process based on a random sample from the underlying copula. These results are the first to extend the findings of Genest and Segers [11] beyond the bivariate case, except at independence.

Because Theorem 1 reduces to Proposition 1 in [11] when $d=2$, it is obvious from the discussion therein that the conditions identified here cannot possibly be necessary. While the conditions are sufficient to encompass some non-trivial multivariate copulas, as shown in Examples 1-2, it is unclear whether inequality (3) generally fails for Archimedean and elliptical copulas, or whether and how the conditions identified herein could be weakened. This issue is left for future research.

Acknowledgments: This research project was supported by grants from the Canada Research Chairs Program and the Natural Sciences and Engineering Research Council of Canada (CG: RGPIN/04720-2016; MM: RGPIN/06536-2018; JGN: RGPIN/067801-2015).

\section{References}

[1] Avérous, J. and J.-L. Dortet-Bernadet (2004). Dependence for Archimedean copulas and aging properties of their generating functions. Sankhyā 66(4), 607-620.

[2] Bingham, N.H., C.M. Goldie, and J.L. Teugels (1989). Regular Variation. Cambridge University Press.

[3] Charpentier, A. and J. Segers, (2009). Tails of multivariate Archimedean copulas. J. Multivariate Anal. 100(7), $1521-1537$. 
[4] Colangelo, A., M. Scarsini, and M. Shaked (2005). Some notions of multivariate positive dependence. Insurance Math. Econom. 37(1), 13-26.

[5] Cramér, H. (1946). Mathematical Methods of Statistics. Princeton University Press.

[6] Denuit, M., J. Dhaene, M.J. Goovaerts, and R. Kaas (2005). Actuarial Theory for Dependent Risks: Measures, Orders and Models. Wiley, New York.

[7] Drouet-Mari, D. and S. Kotz (2001). Correlation and Dependence. Imperial College Press, London.

[8] Fang, K.T., S. Kotz, and K.W. Ng (1990). Symmetric Multivariate and Related Distributions. Chapman \& Hall, London.

[9] Genest, C., A.-C. Favre, J. Béliveau, and C. Jacques (2007). Metaelliptical copulas and their use in frequency analysis of multivariate hydrological data. Water Resources Res. 43, W09401, 12 pp.

[10] Genest, C., J.G. Nešlehová, and B. Rémillard (2017). Asymptotic behavior of the empirical multilinear copula process under broad conditions. J. Multivariate Anal. 159, 82-110.

[11] Genest, C. and J. Segers (2010). On the covariance of the asymptotic empirical copula process. J. Multivariate Anal. 101(8), 1837-1845.

[12] Jaser, M., S.Haug, and A. Min (2017). A simple non-parametric goodness-of-fit test for elliptical copulas. Depend. Model. 5, 330-353.

[13] Joe, H. (2014). Dependence Modeling With Copulas. CRC Press, Boca Raton FL.

[14] Kotz, S., N. Balakhrishnan, and N.L. Johnson (2000). Continuous Multivariate Distributions: Models and Applications. Second edition. New York, Wiley.

[15] Larsson, M. and J. Nešlehová (2011). Extremal behavior of Archimedean copulas. Adv. Appl. Probab. 43(1), 195-216.

[16] Lehmann, E.L. (1966). Some concepts of dependence. Ann. Math. Statist. 37(5), 1137-1153.

[17] Mai, J.F. and M. Scherer (2014). Financial Engineering with Copulas Explained. Palgrave MacMillan, London.

[18] McNeil, A.J., R. Frey, and P. Embrechts (2015). Quantitative Risk Management: Concepts, Techniques and Tools. Revised edition. Princeton University Press.

[19] McNeil, A.J. and J. Nešlehová (2009). Multivariate Archimedean copulas, $d$-monotone functions and $\ell_{1}$-norm symmetric distributions. Ann. Statist. 37(5B), 3059-3097.

[20] Nelsen, R.B. (2006). An Introduction to Copulas. Second edition. Springer, New York.

[21] Salvadori, G., C. De Michele, N.T. Kottegoda, and R. Rosso (2007). Extremes in Nature: An Approach Using Copulas. Dordrecht, The Netherlands.

[22] Segers, J. (2012). Asymptotics of empirical copula processes under non-restrictive smoothness assumptions. Bernoulli 18(3), 764-782.

[23] Shorack, G.R. and J.A. Wellner (1986). Empirical Processes With Applications to Statistics. Wiley, New York.

[24] Sklar, A. (1959). Fonctions de répartition à $n$ dimensions et leurs marges. Publ. Inst. Statist. Univ. Paris 8, 229-231.

[25] Wang, X. and J. Yan (2013). Practical notes on multivariate modeling based on elliptical copulas. J. SFdS 154(1), 102-115. 